\title{
Fatal Cerebral Infarction in an Asymptomatic Young Patient With Primary Antiphospholipid Syndrome
}

\author{
Shinjiro Muneta, M.D., Eisuke Yokota, M.D., Seiichiro Watanabe, M.D., \\ Isao Matsumoto, M.D. and Yoriaki Yamashita, M.D*
}

\begin{abstract}
An 18-year-old woman with primary antiphospholipid syndrome developed a major cerebral infarction leading to brain death despite intensive treatment with steroids, urokinase, glyceol and heparin. Fatal strokes associated with this syndrome are rare. A computed tomographic scan of the brain suggested occlusion of the main trunk of the right middle cerebral artery. The titer of antibodies against cardiolipin/ $\beta_{2}$-glycoprotein I complex in serum was extremely high.
\end{abstract}

(Jpn Circ J 1995; 59: 641-645)

A

NTIPHOSPHOLIPID antibodies (aPL) are frequently detected in patients with systemic lupus erythematosus (SLE) or related autoimmune diseases. The main clinical complications associated with the presence of these antibodies are vascular occlusions, recurrent fetal loss and thrombocytopenia. The term "antiphospholipid syndrome" has been applied to patients characterized by these pathologic features associated with increased levels of aPL1,2 We report here a young woman with primary antiphospholipid syndrome who developed major cerebral infarction leading to brain death. The clinical significance of aPL and the management of patients with aPL are also discussed.

\section{CASE REPORT}

An 18-year-old woman developed chilblains on her feet in January 1989. Since her left 4th toe became gangrenous, she underwent amputation of the left 4 th toe in

Key words:

Anticardiolipin antibodies

Antiphospholipid antibodies

$\beta_{2}$-glycoprotein I

Fatal cerebral infarction

Brain death
January 1990 at our hospital. Serological tests at that time gave a false-positive serologic test for syphilis, positive antinuclear antibody (ANA) and a positive LE test, but there were no clinical signs suggestive of connective tissue diseases such as SLE. Over the next 3 years, she experienced relatively good health without any thrombotic events until February 24, 1993, when she was readmitted to our hospital because of a sudden onset of dysarthria and left hemiplegia.

On admission, her blood pressure was $154 / 76 \mathrm{mmHg}$, her pulse rate was $60 / \mathrm{min}$ and regular, and her body temperature was 37.1 ${ }^{\circ} \mathrm{C}$. There were no abnormal physical findings in the heart, lungs or abdomen. She was not completely alert and had difficulty in speaking, but was well-oriented and responded normally to questions and commands. Conjugate deviation to the right and right-sided homonymous hemianopsia were present. Left flaccid hemiparesis with hypesthesia was observed. The deep tendon reflexes of the extremities were equal and normoactive, but Babinski sign was positive on the left. Hematological examination showed a mild thrombocytopenia $\left(10.8 \times 10^{4}\right.$

(Received August 4, 1994; accepted March 2, 1995)

Department of Internal Medicine and ${ }^{*}$ Department of Neurology, Matsuyama Red Cross Hospital, Matsuyama, Ehime 790, Japan

Mailing address: Shinjiro Muneta, M.D., Department of Internal Medicine, Matsuyama Red Cross Hospital, Matsuyama, Ehime 790, Japan 


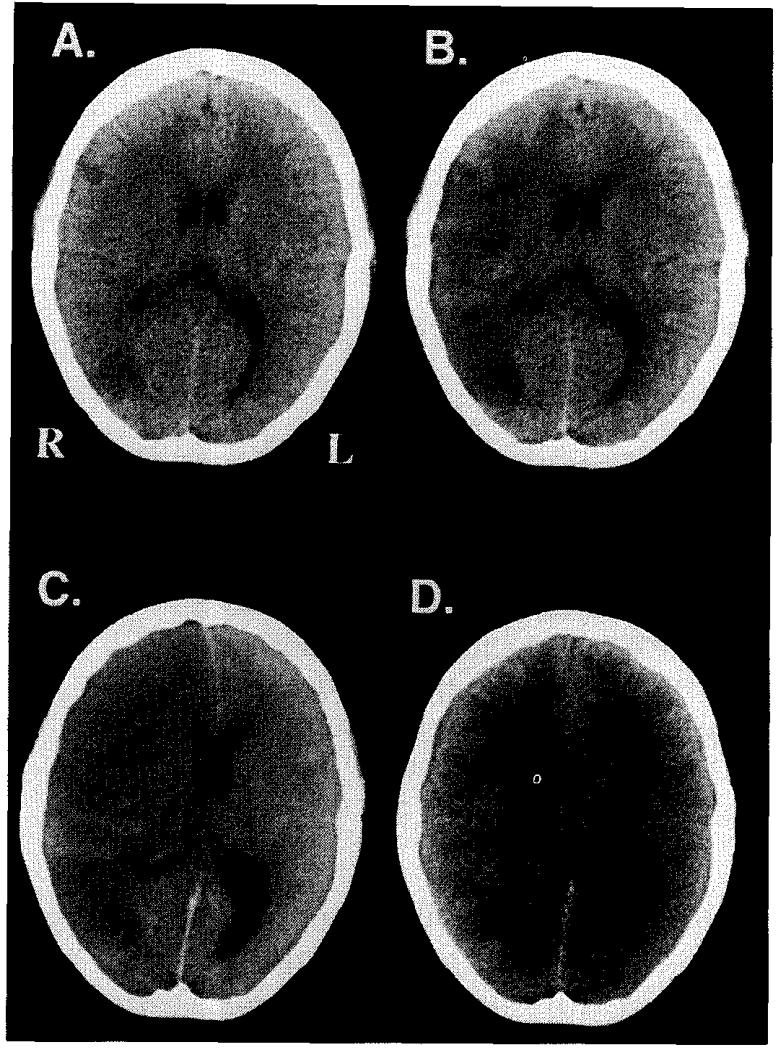

Fig.1. Plain computed tomographic (CT) scans of the brain at the level of the lateral ventricle.
A. On admission ( $2 \mathrm{~h}$ after onset) (1993. 2. 24),
B. The 2nd hospital day (1993. 2. 25),
C. The 6th hospital day (1993. 3. 1),
D. 19 weeks after onset (1993. 7. 8).

$/ \mathrm{mm}^{3}$ ). Blood chemistry findings, including liver function, renal function, serum electrolytes, serum lipids levels and fasting blood glucose, were within normal limits. The erythrocyte sedimentation rate was $17 \mathrm{~mm} / \mathrm{h}$ and $\mathrm{C}$-reactive protein was negative. Immunologic tests showed a false-positive rapid plasma reagin (RPR) test for syphilis (Chemo-Sero-Therapeutic Research Institute, Kumamoto, Japan), a positive LE test, positive ANA with a speckled pattern and positive anti-single stranded DNA antibody (a-ssDNA), whereas anti-double stranded DNA antibody (a-dsDNA), anti-RNP antibody, anti-Sm antibody and rheumatoid factor were negative. CH50 was slightly decreased $(30 \mathrm{U} / \mathrm{ml})$, but serum levels of complement components $(\mathrm{C} 3, \mathrm{C} 4)$ were within normal limits. The titers of anticardiolipin antibodies (aCL) were mildly elevated; IgG at 4.8 (normal range: $<1.0$ ) and $\operatorname{IgM}$ at 1.2 (normal range: $<1.0$ ). The titer of antibodies to cardiolipin (CL)/ $\beta_{2^{-}}$ glycoprotein I ( $\beta_{2}$-GPI) complex was markedly elevated $(>125 \mathrm{U} / \mathrm{ml}$, normal range: $<3.5$ ) (optical density at $450 \mathrm{~nm}$ was 1.283 in the presence of $\beta_{2}$-GPI and 0.537 in the absence of $\beta_{2}$-GPI) according to the ELISA method developed by Yamasa Shoyu Co. Ltd? Circulating immune complexes $(\mathrm{C} 1 \mathrm{q}$ binding assay) were negative. APTT was within the normal range $(36.9 \mathrm{sec}$, normal range: $26.0-38.0 \mathrm{sec}$ ). Chest $\mathrm{X}$-ray film and electrocardiogram showed no abnormalities. Echocardiogram did not reveal endocarditis, valvulitis or cardiac thrombus. Plain computed tomographic (CT) scan of the brain taken $2 \mathrm{~h}$ after the onset was almost normal except for a focal, low-density area just behind the posterior horn of the right lateral ventricle (Fig. 1A).

The clinical course is shown in Fig. 2. Since her neurological signs suggested cerebral thrombosis, immediately after admission she received steroid pulse therapy, urokinase and heparin. A CT scan of the brain on the second hospital day showed a large, heterogeneous, low-density area in the right cerebral hemisphere which corresponded to the territory of the right middle cerebral artery (MCA) (Fig. 1B). Despite intensive treatment, $C T$ scans of the brain disclosed progressive worsening of the brain edema. On the fourth hospital day, respiratory arrest developed abruptly and artificial respiration was started. On the 6th day, she was diagnosed as brain death based on deep coma, apnea, bilateral dilated pupils, absence of brainstem reflex, a flat electroencephalogram and absence of auditory evoked brainstem response. Plain CT scan of the brain on the 6th day showed a widespread, low-density area in the right half of the brain with marked compression and shift of the lateral ventricles (Fig. 1C). The loss of all cisterns and subarachnoid space suggested cerebral and cerebellar herniation. Despite the absence of brainstem function, she survived for about 6 months while being treated with prednisolone $(20 \mathrm{mg} /$ day $)$, catecholaminergic drugs, vasopressin and heparin under the support of artificial ventilation and parenteral hyperalimentation. Plain CT scan of the brain 19 weeks after the onset showed a diffuse, homogenous, lowdensity area over the whole brain (Fig. 1D). 


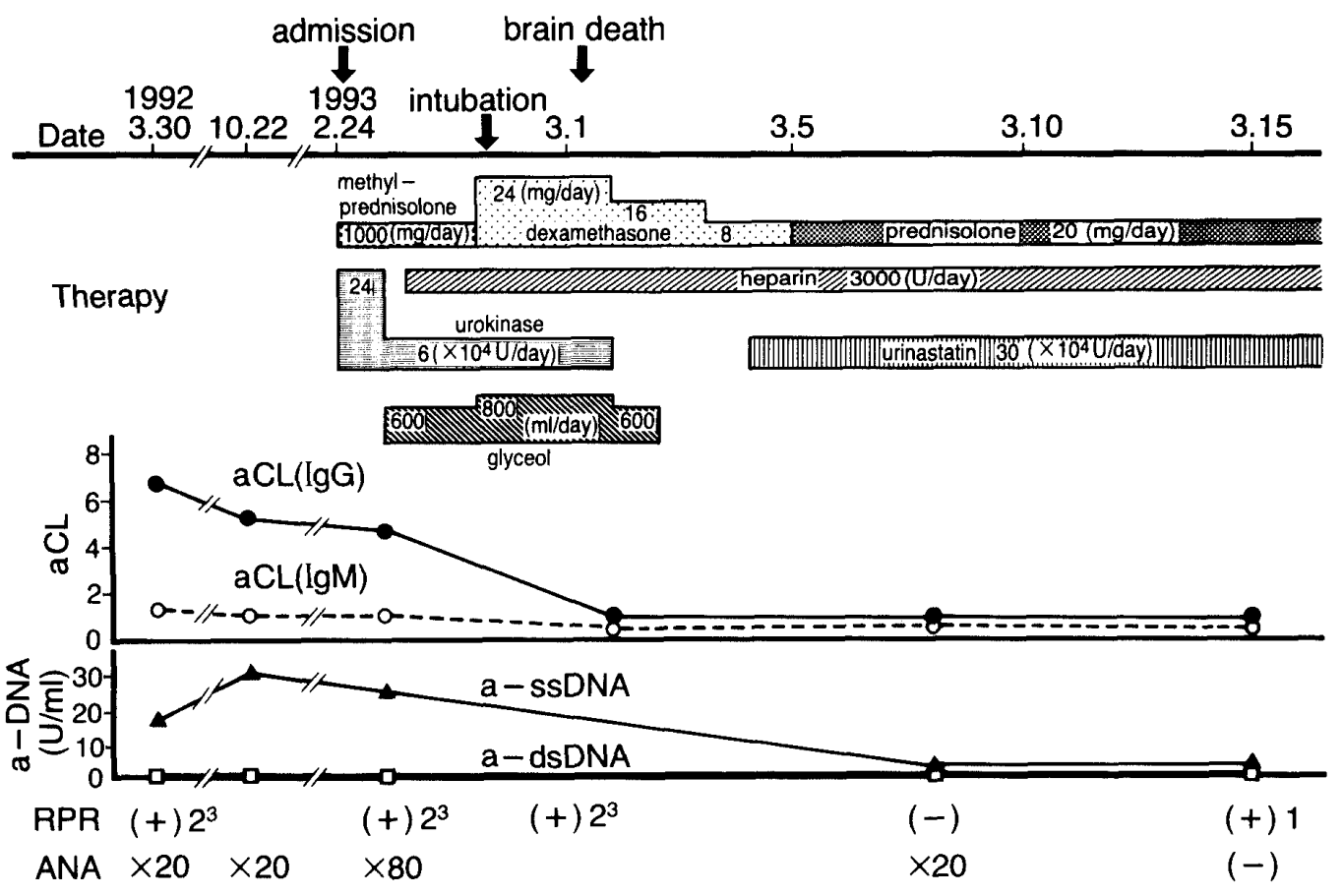

Fig.2. Clinical course.

Immediately after admission, the patient was given steroid pulse therapy (methylprednisolone sodium succinate $1,000 \mathrm{mg} /$ day for three days) and urokinase $(24 \times$ $10^{4} \mathrm{U} /$ day on the first day and then $6 \times 10^{4} \mathrm{U} /$ day for 6 days) with a combination of heparin and glyceol. After steroid pulse therapy, dexamethasone was administered for 7 days, and then prednisolone was continued at a daily maintenance dose of $20 \mathrm{mg} /$ day until her death. Urinastatin was transiently administered to prevent disseminated intravascular coagulation. aCL: anticardiolipin antibody, adsDNA: anti-double stranded DNA antibody, a-ssDNA: anti-single stranded DNA antibody, RPR: rapid plasma reagin (serologic test for syphilis), The numbers in the RPR column indicate the maximum serum dilution rate with positive RPR. ANA: antinuclear antibody.

She died of cardiac arrest on August 19, 1993.

Chronological changes in the titers of autoantibodies are shown in Fig. 2. The increased titers of aCL (IgG, IgM) were normalized within a week after beginning steroid therapy. The titers of a-ssDNA, ANA and RPR were also normalized within 1 month. These autoantibodies remained negative or at a low level over the clinical course until her death. The titers of aCL, assDNA, ANA and RPR on admission were almost the same as those at the outpatient clinic. The titer of a-dsDNA remained negative.

\section{DISCUSSION}

Our patient was considered to be diagnosed as primary antiphospholipid syndrome, since she had a history of arterial thrombosis (gangrene of the toe) and showed a persistent elevation of aCL titers without apparent clinical features of SLE or lupus-like disease.

Her present stroke was attributed to arterial thrombosis related to aCL, since she had no other risk factors for cerebrovascular disease, and echocardiogram revealed no vegetation or mural thrombus. A CT scan of the brain suggested cerebral infarction due to occlusion of the main trunk of the right MCA or the right internal carotid artery. Levine et $\mathrm{al}^{4}$ reported that cerebral angiography revealed large-vessel occlusion or stenosis without changes which might suggest vasculitis in some patients with aPL who presented with cerebral or visual dysfunction. Strokes associated with aPL are often recurrent, multiple and are caused by occlusion of relatively large cerebral arteries, but are rarely fatal, 5 Fatal major cerebral infarction leading to brain death in primary antiphospholipid syndrome has not yet been 
reported.

aPL can be detected in blood samples not only in SLE and related autoimmune diseases, but also in syphilis and other various infectious diseases. Recent studies showed that aCL associated with autoimmune diseases recognize a novel epitope generated by the interaction of phospholipids and a cofactor, whereas aCL associated with infectious diseases bind to phospholipids directly6 This cofactor was identified as $\beta_{2}$-GPI (apolipoprotein $\mathrm{H}$ ) by McNeil et al? Matsuura et al 6,8 showed that the addition of human cofactor enhanced the aCL titer in SLE and reduced it in syphilis, and suggested that autoimmune-type aCL can be discriminated from infectious-type aCL. In our patient, the aCL titer, expressed in terms of optical density, was markedly increased by the addition of $\beta_{2}$-GPI, which suggests the existence of autoimmune-type aCL. Recent evidence indicates that $\beta_{2}$-GPI exerts multiple inhibitory effects on blood coagulation?-11 These findings raise the possibility that aPL interfere with $\beta_{2}$-GPI function, thereby predisposing the patient to thrombotic diathesis. The titer of antibodies to $\mathrm{CL} / \beta_{2}$-GPI complex is considered to be a more useful predictor of thrombosis than the titer of antibodies to CL itself. The extremely high titer of antibodies to $\mathrm{CL} / \beta_{2}$-GPI complex in our patient may be attributed to the present fatal cerebral infarction.

It is still unclear how to prevent vascular occlusions in primary antiphospholipid syndrome. Our patient had been untreated until the present stroke because she showed low positive aCL levels and had no clinical or laboratory evidence of active connective tissue diseases. Although the aCL level is a useful predictor of vascular thrombosis 12 an increase from a low to a high positive aCL titer can not be used to predict the onset of thromboembolic events. ${ }^{13,14}$ Our patient also showed no rise in titers of aCL, a-ssDNA, ANA or RPR preceding the present event. It has been recently recommended that asymptomatic patients with sustained high levels of aCL should receive long-term, lowdose aspirin therapy, and patients with one or more episodes of vascular occlusion should receive long-term treatment with oral anticoagulants such as warfarin, even if the other episodes occurred many years previ- ously?

We should recognize that patients with antiphospholipid syndrome, even asymptomatic young patients, may develop fatal strokes, and therefore require strict management and long-term follow-up to prevent serious occlusive vascular complications associated with aPL. Methods for preventing and predicting vascular complications are needed, especially in asymptomatic young patients with this syndrome.

\section{REFERENCES}

1. Hughes GRV, Harris EN, Gharavi AE: The antiphospholipid syndrome. J Rheumatol 1986; 13: 486-489

2. Asherson RA: Antiphospholipid antibodies and syndromes. In: Lahita RG, editor. Systemic Lupus Erythematosus. New York: Churchill Livingstone $1992 ; 587-635$

3. Yamamoto $\mathrm{T}$, Yoshimura $\mathrm{S}$, Genshi $\mathrm{Y}$, et al: Measurement of antiphospholipid antibody by ELISA using purified $\beta_{2}$-glycoprotein I in preeclampsia. Clin Exp Immunol 1993; 94: 196-200

4. Levine SR, Deegan MJ, Futrell N, Welch KMA: Cerebrovascular and neurologic disease associated with antiphospholipid antibodies: 48 cases. Neurology 1990; 40: $1181-1189$

5. Asherson RA, Khamashta MA, Gil A, et al: Cerebrovascular disease and antiphospholipid antibodies in systemic lupus erythematosus, lupus-like disease, and the primary antiphospholipid syndrome. Am J Med 1989; 86: 391-399

6. Matsuura E, Igarashi Y, Fujimoto M, et al: Heterogeneity of anticardiolipin antibodies defined by the anticardiolipin cofactor. J Immunol 1992; 148: 3885-3891

7. McNeil HP, Simpson RI, Chesterman CN, Krilis SA: Anti-phospholipid antibodies are directed against a complex antigen that includes a lipidbinding inhibitor of coagulation: $\beta_{2}$-glycoprotein I (apolipoprotein H). Proc Natl Acad Sci USA 1990; 87: $4120-4124$

8. Matsuura E, Igarashi Y, Fujimoto M, Ichikawa K, Koike T: Anticardiolipin cofactor(s) and differential diagnosis of autoimmune disease. Lancet 1990; 336: $177-178$

9. Schousboe I: $\beta_{2}$-Glycoprotein I: A plasma inhibitor of the contact activation of the intrinsic blood coagulation pathway. Blood 1985; 66: 1086-1091

10. Nimpf $\mathbf{J}$, Wurm $\mathbf{H}$, Kostner GM: $\beta_{2}$-Glycoprotein I (apo-H) inhibits the release reaction of human platelets during ADP-induced aggregation. Atherosclerosis 1987; 63: 109-114

11. Nimpf J, Bevers EM, Bomans PH, et al: Prothrombinase activity of human platelets is inhibited by $\beta_{2}$-glycoprotein I. Biochim Biophys Acta 1986; 884: $142-149$

12. Harris EN, Chan JKH, Asherson RA, et al: Thrombosis, recurrent fetal loss, and thrombocytopenia: predictive value of the anticardiolipin

Japanese Circulation Journal Vol.59, September 1995 
antibody test. Arch Intern Med 1986; 146: 2153-2156

13. Asherson RA, Baguley E, Pal C, Hughes GRV: Antiphospholipid syndrome: five year follow up. Ann Rheum Dis 1991; 50: 805-810
14. Out JH, De Groot PG, Hasselaar P, van Vliet M, Derksen RHWM: Fluctuations of anticardiolipin antibody levels in patients with systemic lupus erythematosus: a prospective study. Ann Rheum Dis 1989; 48: 1023-1028 